This is an author produced version of a paper published in Inorganic Chemistry.

This paper has been peer-reviewed but may not include the final publisher proof-corrections or pagination.

Citation for the published paper:

Andreas O. Tirler, Ingmar Persson, Thomas S. Hofer and Bernd M. Rode. (2015) Is the Hexacyanoferrate(II) Anion Stable in Aqueous Solution? A Combined Theoretical and Experimental Study. Inorganic Chemistry. Volume: 54, Number: 21, pp 10335-10341.

http://dx.doi.org/10.1021/acs.inorgchem.5b01701.

Access to the published version may require journal subscription.

Published with permission from: American Chemical Society.

Standard set statement from the publisher:

"This document is the Accepted Manuscript version of a Published Work that appeared in final form in Inorganic Chemistry, copyright (C) American Chemical Society after peer review and technical editing by the publisher. To access the final edited and published work see http://dx.doi.org/10.1021/acs.inorgchem.5b01701."

Epsilon Open Archive http://epsilon.slu.se 


\section{Is the Hexacyanoferrate(II) Anion Stable in Aqueous Solution? \\ A Combined Theoretical and Experimental Study}

Andreas O. Tirler ${ }^{1)}$, Ingmar Persson ${ }^{2)}$, Thomas S. Hofer ${ }^{1)}$ and Bernd M. Rode ${ }^{1) *}$

1) Theoretical Chemistry Division

Institute of General, Inorganic and Theoretical Chemistry University of Innsbruck, Innrain 80-82, A-6020 Innsbruck, Austria

E-Mail: Bernd.M.Rode@uibk.ac.at Tel.: +43-512-507-57160

2) Department of Chemistry and Biotechnology

Swedish University of Agricultural Sciences

P.O. Box 7015, SE-750 07 Uppsala, Sweden

E-mail: ingmar.persson@slu.se Tel.: +46-18-671547

July 9,2015

${ }^{*}$ Corresponding author 


\begin{abstract}
A combined theoretical and experimental study has been performed to elucidate the structural and dynamical properties of aqueous hexacyanoferrate(II) isolated and in presence of potassium ions. It is shown that in absence of counter ions, the highly negatively charged hexacyanoferrate(II) complex is not stable in aqueous solution. However, if the high negative charge is compensated by potassium counter ions, a stable complex is observed, which is proven by theoretical simulations as well as by EXAFS experiments. From the simulation it is found that potassium ions surrounding the complex are highly mobile and thus, cannot be observed in the experiment. The structure of aqueous hexacyanoferrate(II) in presence of potassium ions is identical to that of the solid state structure, but the mobility of potassium ions is significantly increased in the liquid. These highly mobile potassium ions circulating the complex should be the reason for the very short hydrogen bond lifetimes in the femtosecond range of the cyanide ligands.
\end{abstract}




\section{Introduction}

Because of their outstanding physico-chemical properties cyanoferrates are used in many industrial processes as well as in daily life. Their pronounced photo-induced magnetization effect makes them very popular for the use in magnetic devices, ${ }^{1-5}$ and they are also wellknown for their sensing capabilities ${ }^{6-9}$ and in ion-sieving membranes, ${ }^{10}$ electrocatalysis, ${ }^{11}$ corrosion protection ${ }^{12}$ and charge storage. ${ }^{13}$ Furthermore, they are used in the photographic, mining and metallurgical industry, thus requiring arrangements for a safe and economical treatment. ${ }^{14}$ The manifold applications of cyanoferrates need a proper characterization of their structural and dynamical behaviour under the applied conditions, often corresponding to those in aqueous media. A better understanding of structural entities can significantly contribute to tune the physico-chemical properties and hence, to improve the respective processes.

Among the huge number of structurally known cyanoferrates, hexacyanoferrates are probably the most important and, therefore, the most extensively studied ones. This can also be seen from the numerous reported experimental investigations of hexacyanoferrates in the past. ${ }^{14-30}$ According to the literature hexacyanoferrate(II) ions, $\left[\mathrm{Fe}(\mathrm{CN})_{6}\right]^{4-}$, are octahedral coordination complexes, which, depending on $\mathrm{pH}$ and cyanide concentration, tend to dissociate upon heat or light exposure ${ }^{14,15,19,31}$ or in presence of mercury(II), ${ }^{28}$ silver(I), ${ }^{24,28}$ palladium(II) ${ }^{28}$ and gold(III) ${ }^{28}$ as they form even stronger complexes with cyanide than iron(II) and iron(III). A degradation of hexacyanoferrates by microorganisms has also been reported. ${ }^{19,20}$ The resulting species is a pentacyanoferrate(II) $\left[\mathrm{Fe}(\mathrm{CN})_{5}\right]^{3-}$ 14,15,24,27,28 or a tetracyanoferrate(II) $\left[\mathrm{Fe}(\mathrm{CN})_{4}\right]^{2-} \cdot \mathrm{H}_{2} \mathrm{O} .{ }^{21}$ However, an important issue is the effect of counter-ions on the coordination behaviour of highly charged complexes. It is well known that counter-ions may stabilize coordination polyhedra. ${ }^{32-34}$ Studies on $\mathrm{SiO}_{4}^{4-}$ (unpublished) and $\left[\mathrm{UO}_{2}\left(\mathrm{CO}_{3}\right)_{3}\right]^{4-}{ }^{35}$ for instance have clearly shown that in absence of proper counter-ions four-fold negatively charged anions are not stable in aqueous solution and as they immediately undergo reactions towards a lower charged entity. Therefore, in the case of the anion $\left[\mathrm{Fe}^{I I}(\mathrm{CN})_{6}\right]^{4-}$ it is questionable whether a four-fold negatively charged ion is really stable in a dilute aqueous solution, since this would be the first stable complex of this kind so far. It seemed interesting, therefore, to investigate the structure of the $\left[\mathrm{Fe}^{I I}(\mathrm{CN})_{6}\right]^{4-}$ ion in water without the presence of counter-ions. Theoretical investigations have the advantage to provide structural and dynamical data of hydrated complexes without counter-ions and at very low concentrations. Therefore, processes beyond the experimental limit can be analyzed and fundamental information for the interpretation of ex- 
perimentally observed phenomena can be obtained. The present study aimed at elucidating structural details of the sole hexacyanoferrate $\left[\mathrm{Fe}(\mathrm{CN})_{6}\right]^{4-}$ ion and in presence of counterions, potassium in this study, in aqueous solution using quantum mechanical/molecular mechanical (QM/MM) simulations at different levels of theory and by comparing them to extended X-ray absorption fine spectroscopic (EXAFS) data in both solid state and aqueous solution.

\section{Computational Methods}

In the present work a hybrid quantum mechanical/molecular mechanical (QM/MM) simulation technique was used. ${ }^{36} \mathrm{QM} / \mathrm{MM}$ approaches are very popular because they combine the accuracy of QM and the efficiency of MM in one single framework. Thereby, the system's chemically most relevant region, which often corresponds to a solvated species, is treated by a proper QM method, which is embedded in an MM treated region to mimic a physically reasonable solvent environment. An important concern of hybrid simulation techniques is the QM/MM coupling between the two regions to avoid any discontinuities in energies and associated forces. Suitable solute-solvent potentials are therefore required. However, when treating highly charged ions accurate potential functions are difficult, or in some cases even impossible, to obtain. Therefore, an improved QM/MM simulation technique was developed, referred to as quantum mechanical charge field (QMCF) approach. ${ }^{37-40}$ The main advantage is that the need for solute-solvent potentials is avoided by enlarging the QM region and by subdividing it into two subregions denoted as QM core and QM layer. It has been shown that by chosing an adequate size of the QM region, the QM layer represents a proper buffer region between the QM core and the MM region and thus, the typically short-ranged non-Coulombic solute-solvent interactions can be safely neglected. ${ }^{39,40}$ Therefore, QMCF enables a general application for studying a broad spectrum of chemical systems on a QM/MM level and was already successfully used in the investigations of a large number of different species in solution. ${ }^{35,41-44}$

A critical issue when employing QM approaches is the choice of an adequate level of theory. Ab initio Hartree-Fock (HF) does not include electron correlation, but is has been shown that simulation data obtained for hydrated ions by using HF is in excellent agreement with experimental results, thus indicating that the neglect of electron correlation might not play a significant role for obtaining correct structural and dynamical results. ${ }^{35,41-44}$ Furthermore, the computational cost offers an optimal compromise between accuracy and 
computational demand. Density Functional Theory (DFT) on the other hand incorporates electron correlation to some extent due to its semiempirical formalism, but it is not possible to evaluate the amount of electron correlation considered by the chosen functional and thus, no control of the respective contribution is given. Nevertheless, DFT methods are very popular and widely used, because correct results are obtained with a reasonable computational effort. Especially hybrid DFT functionals such as B3LYP are valuable approaches, because the critical exchange term is calculated exactly and electron correlation is taken into account by an approximate correlation functional only moderately increasing the computational time. A more sophisticated ab initio QM method is second order Møller-Plesset perturbational theory (MP2). The HF solution is thereby supplemented by an additional electron correlation term using perturbational theory. The obtained results are thus more accurate, but for large numbers of atoms computational costs increase dramatically. However, it is possible to run MD simulations applying resolution of identity second order Møller-Plesset perturbational theory (RI-MP2), ${ }^{45-47}$ which gives results of similar quality reducing at the same time the computational demand. Such MD simulations are still very time-consuming and simulation times of only a few picoseconds can be obtained. Therefore RI-MP2 data is used to qualitatively evaluate results obtained by MD simulations at a lower level of theory such as HF and B3LYP.

In this study the structure of the sole hydrated hexacyanoferrate(II) anion $\left[\mathrm{Fe}(\mathrm{CN})_{6}\right]^{4-}$ and in presence of potassium ions are investigated via QMCF-MD simulations applying different levels of theory, namely HF, B3LYP and RI-MP2 and the obtained results are compared to EXAFS data as well as to other data reported in the literature.

The MD simulations were performed using a cubic water box containing 2500 explicitly treated water molecules applying periodic boundary conditions. The solvent's density was $0.997 \mathrm{~g} / \mathrm{cm}^{3}$ corresponding to a box length of approximately $42 \AA$. The temperature was kept at $298.15 \mathrm{~K}$ employing the Berendsen thermostat. ${ }^{48}$ The Velocity-Verlet algorithm ${ }^{49}$ was used for the time propagation applying a time-step of 0.2 fs for MD simulations using HF and B3LYP and 2.0 fs for those at RI-MP2 level of theory. Coulombic interactions were evaluated within a range of $15.0 \AA$ and the reaction field method ${ }^{50,51}$ was used to correct for the long range nature of electrostatic contributions. The QM core was set to $4.0 \AA$ and included the iron center and the cyanide ligands, the QM layer was set to $7.0 \AA$ including an average of 25 explicitly QM-treated water molecules and the potassium cations in the respective simulation. Thus, a total QM region with a diameter of $14.0 \AA$ was obtained. The associated QM energy and gradient calculations were performed using the TURBO- 
MOLE 6.4 package. For iron center the Stuttgart RSC (relativistic small core) basis set in combination with the respective core-potential ${ }^{52}$ was used. For hydrogen, oxygen, carbon, nitrogen and potassium atoms the respective $6-31 \mathrm{G}(\mathrm{d}, \mathrm{p})$ basis sets ${ }^{53,54}$ were chosen for the HF and RI-MP2 simulations, and the $6-311 \mathrm{G}(\mathrm{d}, \mathrm{p})$ basis sets ${ }^{55,56}$ for the B3LYP simulations. The water-water interactions (for the $\mathrm{QM} / \mathrm{MM}$ coupling and in the MM region) were modeled using the flexible SPC-mTR water model ${ }^{57}$ for the MD simulations applying a time step of 0.2 fs. For the MD simulations using a time step of 2.0 fs water molecules were kept rigid employing the M-SHAKE algorithm. ${ }^{58}$ In the simulations of $\mathrm{K}_{4}\left[\mathrm{Fe}(\mathrm{CN})_{6}\right]$ potassium-water potentials by Reif et al. ${ }^{59}$ were employed. In order to properly relax the degrees of freedom of all particles in the simulation box, both simulated systems were extensively pre-equilibrated by MM-MD simulations before invoking the QM/MM treatment. The $\left[\mathrm{Fe}(\mathrm{CN})_{6}\right]^{4-}$ system was equilibrated for $200 \mathrm{ps}$, whereas the $\mathrm{K}_{4}\left[\mathrm{Fe}(\mathrm{CN})_{6}\right]$ one was subsequently equilibrated for another 100 ps to relax also the potassium-water interactions. Finally the QM/MM treatment was employed as discussed above and trajectories of $15 \mathrm{ps}$ were collected for the respective RI-MP2 simulations, while trajectories of $30 \mathrm{ps}$ were collected for both systems at HF and B3LYP level.

\section{Experimental Methods}

Chemicals: Solid potassium hexacyanoferrate(II) trihydrate, $\mathrm{K}_{4}\left[\mathrm{Fe}(\mathrm{CN})_{6}\right] \cdot 3 \mathrm{H}_{2} \mathrm{O}$, and potassium hexacyanoferrate(III), $\mathrm{K}_{3}\left[\mathrm{Fe}(\mathrm{CN})_{6}\right]$, (both Sigma-Aldrich, $99.99+\%$ purity) were used as purchased.

Aqueous solutions: $0.2 \mathrm{~mol} \cdot \mathrm{dm}^{-3}$ aqueous solutions of $\mathrm{K}_{4}\left[\mathrm{Fe}(\mathrm{CN})_{6}\right]$ and $\mathrm{K}_{3}\left[\mathrm{Fe}(\mathrm{CN})_{6}\right]$ were prepared by dissolving appropriate amounts of salt in $10 \mathrm{ml}$ Millipore filtered water in a measuring flask.

X-ray Absorption Fine Structure (XAFS): The XAFS measurements were performed at the wiggler beam line I811 at MAX-lab, Lund University, which operated at $1.5 \mathrm{GeV}$ and a maximum current of $250 \mathrm{~mA}$. The EXAFS station was equipped with a Si[111] double crystal monochromator. Higher order harmonics were reduced by detuning the second monochromator crystal to reflect $50 \%$ of maximum intensity at the end of the scans at the Fe K absorption edge. The spectrum of an iron foil was recorded simultaneously in transmission mode as reference; the first inflection point of metallic iron is $7111.3 \mathrm{keV} .{ }^{60}$ All data treatment was performed with use of the EXAFSPAK program package. ${ }^{61}$ EXAFS functions were extracted using standard procedures for pre-edge subtraction, spline removal and 
data normalization. ${ }^{62}$ In order to obtain quantitative information the $\mathrm{k}^{3}$-weighted EXAFS oscillations were analyzed by non-linear least squares fitting of the model parameters. All data treatment was made by the use of the EXAFSPAK program package ${ }^{61}$ Model fitting was performed with theoretical phase and amplitude functions including both single and multiple scattering paths using the ab initio code FEFF (version 7.02). ${ }^{63,64}$ Six continuous scans of each sample were run in transmission mode over the energy range of 6960 to 7980 $\mathrm{eV}$ using ion chambers with stationary gas mixtures (nitrogen and helium).

The solid samples were carefully grained and mixed with BN (ca. $30 \mathrm{mg}$ potassium hexacyanoferrate and $50 \mathrm{mg} \mathrm{BN}$ ) and pressed into an $1 \mathrm{~mm}$ thick aluminum frame. 0.2 $\mathrm{mol} \cdot \mathrm{dm}^{-3}$ potassium hexacyanoferrate aqueous solutions were prepared by dissolving the salts in Millipore filtered deionized water. The aqueous solutions were contained in 1.5 $\mathrm{mm}$ thick sample cells made of a Teflon spacer and $6 \mu \mathrm{m}$ thick polypropylene X-ray film as windows and with titanium frames holding the cell together.

\section{Results and Discussion}

\subsection{Species and Structure of sole $\left[\mathrm{Fe}(\mathrm{CN})_{6}\right]^{4-}$ Complexes in Aque- ous Solution}

All three QMCF-MD simulations of $\left[\mathrm{Fe}(\mathrm{CN})_{6}\right]^{4-}$ in aqueous solution were started from the configurations corresponding to the classically pre-equilibrated octahedral arrangement depicted in Fig.1a. However, after invoking the QM/MM treatment and irrespective of the applied QM level, an immediate ligand dissociation forming a pentacyanoferrate(II) $\left[\mathrm{Fe}(\mathrm{CN})_{5}\right]^{3-}$ was observed within the first picosecond of simulation as illustrated in the Fe-C distance plot in Fig.1b. For the pentacyanoferrate(II) a square pyramidal structure is observed, as expected (Fig.1b). Within the second picosecond of simulation a second cyanide dissociation occurred in all three MD simulations and a tetracyanoferrate $\left[\mathrm{Fe}(\mathrm{CN})_{4}\right]^{2-}$ was formed as can be deduced from the Fe-C distance plots in Fig.1c. According to this data obtained at a QM level of theory it can be seen that conventional MM simulations are not suitable for the treatment of such systems, because the inclusion of polarization and charge transfer is essential to properly describe a ligand dissociation. Therefore, it is not surprising that in our preceding MM simulations, and in the MM simulation by Prampolini et al. ${ }^{29}$ the octahedral configuration was maintained, while all MD simulations at a QM level show that two cyanide ligands dissociate within the first two picoseconds of simula- 
tion. This is an important finding evidencing that the isolated four-fold negatively charged anion $\left[\mathrm{Fe}(\mathrm{CN})_{6}\right]^{4-}$ is not stable in aqueous solution, and undergoes ligand dissociation(s) to lower the high negative charge. However, this conclusion seems to be in disagreement with our experimental results as well as with data available in the literature reporting a six-fold octahedral hexacyanoferrate(II) anion in aqueous solution. ${ }^{29,30}$ This discrepancy will be further discussed below. The tetracyanoferrate(II) anion does not expel any more cyanide ligands within the reported simulation time at all three levels of theory. The structure of the tetracyanoferrate(II) shown in Fig.1 is not the stable configuration, and a water molecule enters the coordination sphere to form a five-coordinated square pyramid. The $\left[\mathrm{Fe}(\mathrm{CN})_{4} \mathrm{H}_{2} \mathrm{O}\right]^{2-}$ species undergoes rapid ligand re-orientations within the sampling time of $30 \mathrm{ps}$ (HF and B3LYP) as illustrated in Fig.2a-f. To facilitate the further discussion the ligands were color-coded. In the distinct snapshots taken from the simulation, the various ligand re-orientations can be observed. Especially Fig.2d displays an important species because a square planar configuration of the four cyanide ligands is adopted, which rapidly converts back into a non-planar configuration proving the ultrafast dynamics of the coordination complex. From the pairwise radial distribution functions (RDFs) shown in Fig.3 average iron(II)-ligand bond distances are obtained. The black curves denote RDFs obtained from the HF simulation, red and blue curves RDFs obtained from MD simulations at the B3LYP and RI-MP2 level, respectively. Fig.3a depicts the Fe-C RDFs; an average bond distance of $2.16 \AA$ is obtained in case of HF and $1.89 \AA$ in case of B3LYP and RI-MP2. The significant difference of $0.27 \AA$ between HF and B3LYP or RI-MP2 may partly arise from the neglect of electron correlation by HF with respect to RI-MP2 but also from the smaller basis set used compared to the B3LYP simulation. It is important to stress that these simulation results on a sole $\left[\mathrm{Fe}(\mathrm{CN})_{6}\right]^{4-}$ complex in aqueous solution shall not be compared with simulations and experiments with counter ions present. Fig. $3 \mathrm{~b}$ shows the Fe-N RDFs and the resulting average distances are $3.30 \AA$ in case of HF and $3.08 \AA$ in case of B3LYP and RI-MP2. The difference of $0.22 \AA$ in case of HF versus B3LYP/RI-MP2 is $0.05 \AA$ shorter than for the Fe-C distance indicating that the methodical differences also influence the $\mathrm{C}-\mathrm{N}$ bond and/or geometry of the complex. An additional peak of minor intensity for the HF and B3LYP simulation is observed in Fig.3b. This peak can be assigned to a cyanide ligand temporarily coordinating with the nitrogen atom towards iron(II), and a bond distance of $2.08 \AA$ for HF and $1.98 \AA$ for B3LYP for this species is obtained. For the RI-MP2 simulation no such event is observed, which can be attributed to the shorter simulation time due to the high computational demand of this 
simulation. The Fe-O RDF in Fig.3c shows bond distances of $2.09 \AA$ for HF, $2.07 \AA$ for B3LYP and $1.98 \AA$ for RI-MP2, respectively. It can be concluded that although HF neglects electron correlation, mostly correct structural results are obtained since all relevant species observed at the other levels of theory are seen by applying HF. Additionally it is seen that by using B3LYP in combination with a triple-zeta basis set good quality RI-MP2 data can be achieved for metal-cyanide coordination, but for water coordination B3LYP performs similar to HF deviating approx. $0.1 \AA$ from the bond distance obtained by the RI-MP2 simulation. Apparently the quality of results applying B3LYP depends on the kind of considered ligands. Our results further indicate that electron correlation seems not relevant for hydrated metal ions, but apparently becomes more important in complexes with other ligands than water, in particular those with high electron density such as the cyanide ion.

At this point some important conclusions on the structure of the isolated (hexa)cyanoferrate(II) anion in dilute aqueous solution can be made. MD simulations at different levels of theory do not support a six-fold octahedral coordination reported by experiments. The simulation results clearly indicate that the absence of counter ions strongly affects the chemical properties of highly negatively charged species as $\left[\mathrm{Fe}(\mathrm{CN})_{6}\right]^{4-}$ in aqueous solution. In this particular case the $\left[\mathrm{Fe}(\mathrm{CN})_{6}\right]^{4-}$ complex immediately dissociates two cyanide ligands and picks up a water molecule to form a $\left[\mathrm{Fe}(\mathrm{CN})_{4} \mathrm{H}_{2} \mathrm{O}\right]^{2-}$ complex with square pyramidal configuration where ligand positions are dynamically exchanging on the picosecond time scale. Therefore, it is likely that for very dilute solutions, excluding any influence of counter-ions on the structure, an averaged structure consisting of different configurations as depicted in Fig.2.a-f is observed. Their average structure clearly resembles an octahedral configuration, because all relevant positions of the octahedron are occupied by a cyanide ligand within the measurement period and thus, all scattering signals characteristic for an octahedron are observed. The previously reported theoretical data on the other hand are either QM cluster gas phase calculations based on an optimised single configuration at the B3LYP level $^{29}$ or MM simulations ${ }^{30}$ using a harmonic approach for the modeling of coordinative bonds, thus preventing the observation of ligand dissociations.

\subsection{Structure of $\mathrm{K}_{4}\left[\mathrm{Fe}(\mathrm{CN})_{6}\right]$ in Aqueous Solution}

The structure of the hexacyanoferrate(II) complex has been reported in more than 60 crystallographic studies, Table S1, and some X-ray absorption studies, ${ }^{16-18,31}$ and in aqueous solution ${ }^{18}$ as well. The EXAFS experiments on the hexacyanoferrate(II) and -(III) com- 

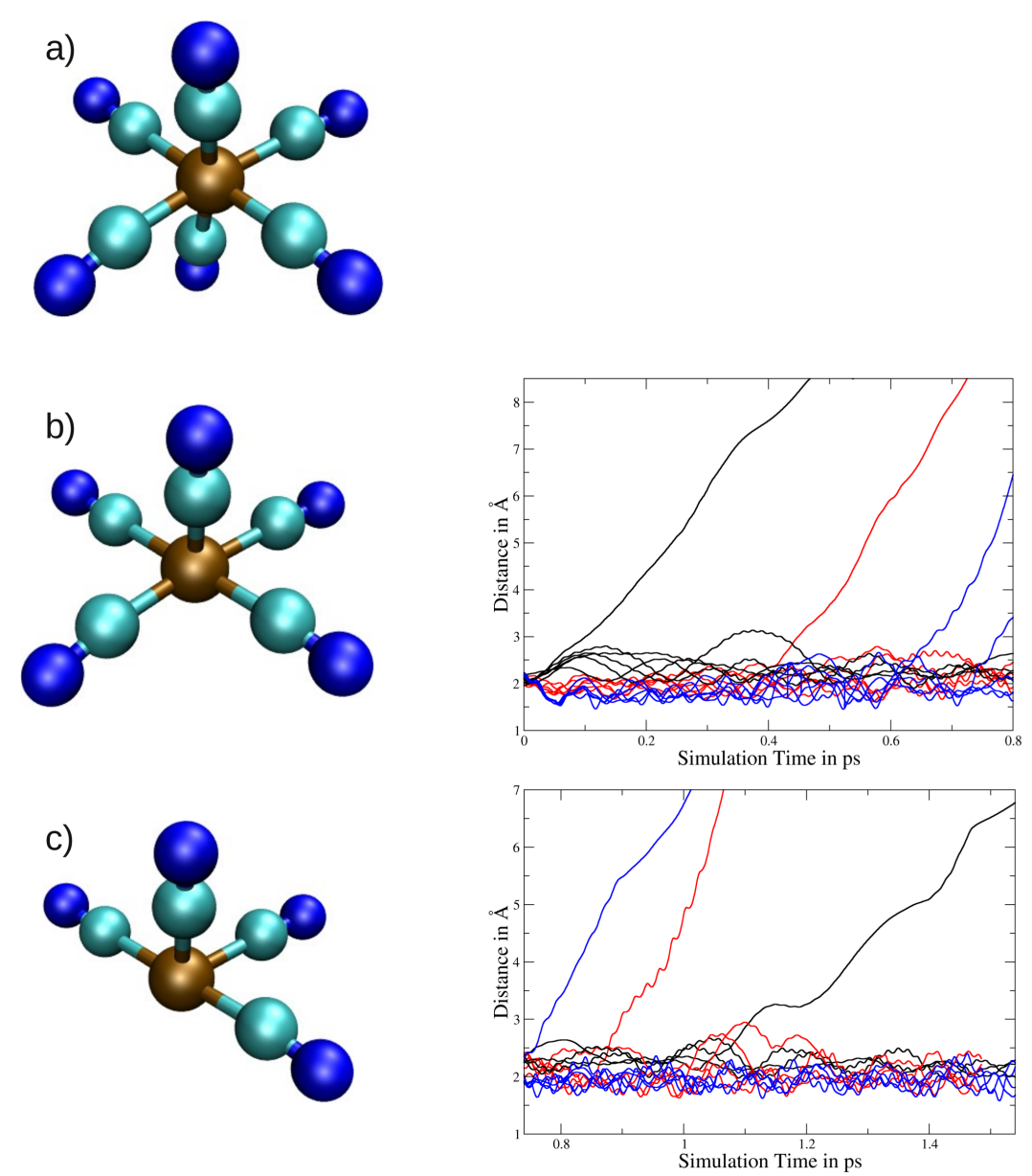

Figure 1: Configurations observed during the dissociation of cyanide ligand visible from Fe-C distance plots: Black graphs correspond to the HF simulation, red and blue graphs to B3LYP and RI-MP2 simulations, respectively. a) The starting configuration obtained from a classical MD simulation. b) The first cyanide ligand has dissociated as shown in the Fe$\mathrm{C}$ distance plot leading to a square pyramidal structure. c) A second ligand dissociation occurs as it can be seen from the $\mathrm{Fe}-\mathrm{C}$ distance plot forming a tetracyanoferrate. The dissociations occurred that fast that no water molecules entered the coordination sphere. 


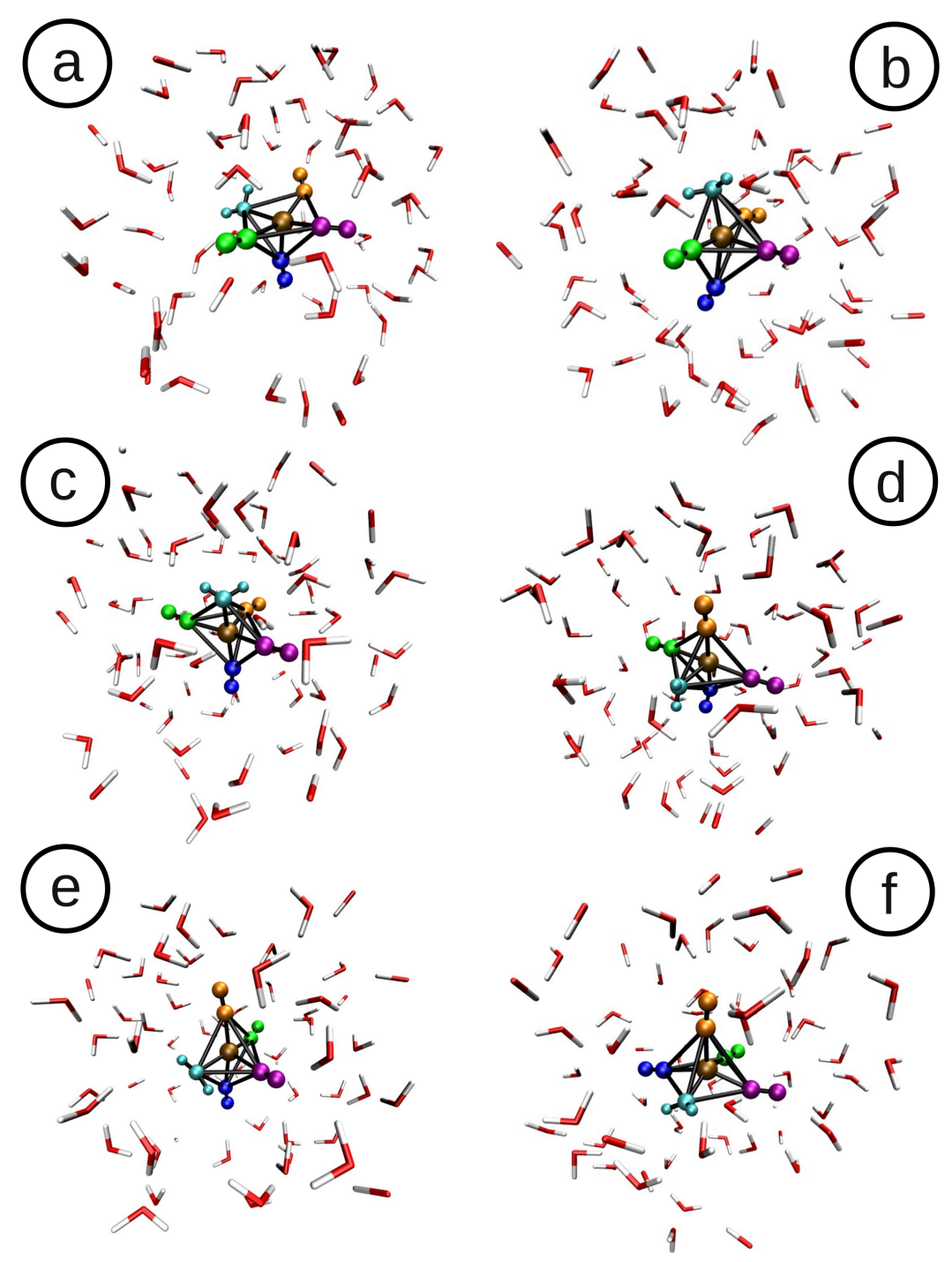

Figure 2: Representative snapshots taken from the MD simulation within $30 \mathrm{ps.} \mathrm{The}$ cyanide ligands of the tetracyanoferrate(II) species with a coordinating water molecule are dynamically changing the positions adopted in the square pyramid: a) the starting snapshot, where the water molecule enters the coordination sphere of iron(II). b) The water molecule (cyan) changes into the apical position. c) A cyanide ligand (green) changes into another equatorial coordination site. d) A cyanide (orange) and the water ligand (cyan) simultaneously change position forming again the square pyramid with four cyanide ligands constituting the basis of the pyramid. e) A cyanide ligand (green) changes coordination site. f) A cyanide ligand (blue) changes position reforming the square pyramid. 


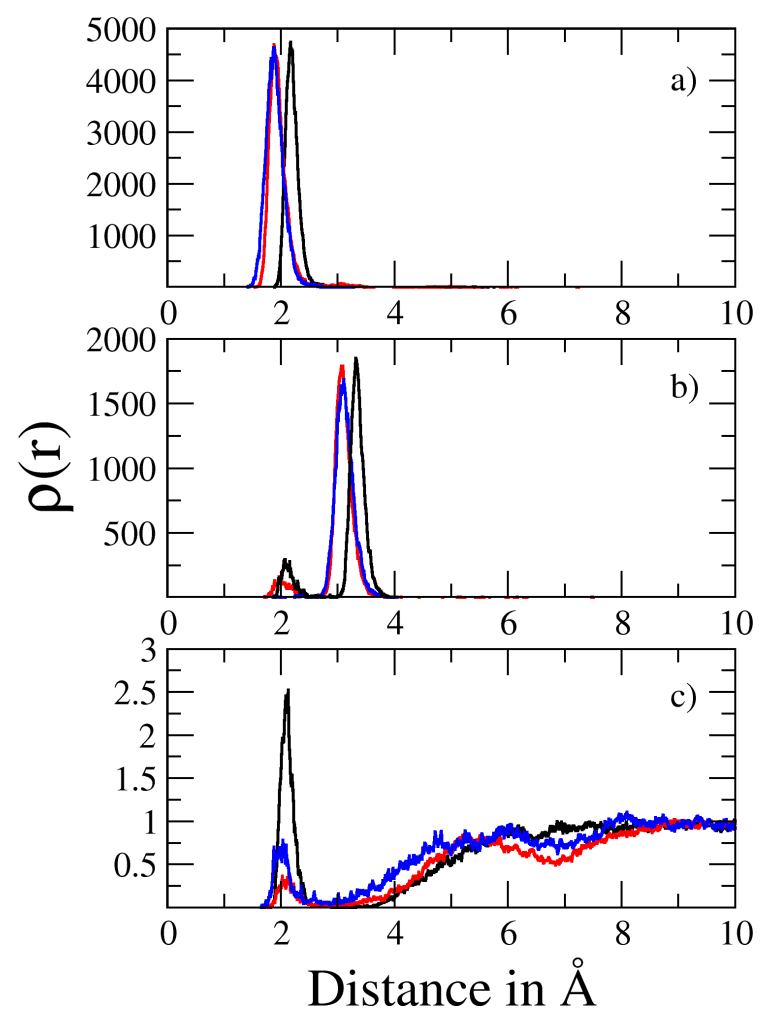

Figure 3: Radial distribution functions (RDFs) for $\left[\mathrm{Fe}(\mathrm{CN})_{4} \mathrm{H}_{2} \mathrm{O}\right]^{2-}$ obtained from QMCF MD simulations at different levels of theory. Black curves denote RDFs obtained from simulations applying HF, red and blue curves denote the corresponding RDFs at the B3LYP and RI-MP2 level. a) Fe-C RDF b) Fe-N RDF c) Fe-O RDF 
plexes in both solid state and aqueous solution have been repeated. The EXAFS data show for both systems that the structure in the solid state and in aqueous solution are identical, and in full agreement with the crystallographic studies and the previous EXAFS study, ${ }^{18}$ Table S1. The only minor difference between the data in solid state and aqueous solution is that Fe-C-N angle is slightly smaller for both systems in solution than in the solids, Table S2, showing the large flexibility of the complex is solution. The structure parameters are summarized in Table S2, and the fit of the experimental data is given in Figures S1 (experimental data) and S2 (Fourier transform). A weakness with structure methods applied on non-crystalline materials, as EXAFS, is that long distances between atoms and with relative large movement relative each other, are very difficult or impossible to observe. It has not been possible to detect the Fe-K distances in the solids where they are known, and this makes it also impossible in aqueous solution where relative movement between Fe and $\mathrm{K}$ is even larger. It is, therefore, very likely that at least some potassium ions are clustered fairly close to the $\left[\mathrm{Fe}(\mathrm{CN})_{6}\right]^{4-}$ complex without being possible to observe by EXAFS; A recent infrared spectroscopic study has shown that water molecules in the hydration shell of the $\left[\mathrm{Fe}(\mathrm{CN})_{6}\right]^{4-}$ complex are more inhomogeneously distributed but more tightly bound to the cyano groups than those of the $\left[\mathrm{Fe}(\mathrm{CN})_{6}\right]^{3-}$ complex. ${ }^{31}$ Those results also suggest that in the hydration shell the $\left[\mathrm{Fe}(\mathrm{CN})_{6}\right]^{4-}$ complex breaks more water structure than the $[\mathrm{Fe}(\mathrm{CN})-6]^{3-}$, which is opposite to the situation of the bulk water region. ${ }^{64,65}$ These results indicate that more potassium ions are clustered around the $\left[\mathrm{Fe}(\mathrm{CN})_{6}\right]^{4-}$ complex than the $\left[\mathrm{Fe}(\mathrm{CN})_{6}\right]^{3-}$ one. Further MD simulations at the same level of theory were therefore carried out for the potassium hexacyanoferrate(II) complex. Fig.4a shows the hexacyanoferrate(II) complex and the spatial distribution of the four potassium ions in form of a volume map projection obtained from the MD simulations. It is clearly seen that the counter-ions cannot be located at fixed positions, but are circulating around the anion with high speed, thus confirming a highly mobile potassium ion cloud around the hexacyanoferrate(II) complex even in highly dilute solution. Fig.4b depicts Fe-K distance plots. It can be seen that potassium ions are located at distances between approx. 3.5 and $5.8 \AA$ around the iron(II) center, which indicates vigorous dynamics of the counter-ions; the Fe-K distance in solid $\mathrm{K}_{4}\left[\mathrm{Fe}(\mathrm{CN})_{6}\right] \cdot 3 \mathrm{H}_{2} \mathrm{O}$ is in the range 4.20-4.35 $\AA{ }^{66-68}$ It is important to note that the simulated $\mathrm{K}_{4}\left[\mathrm{Fe}(\mathrm{CN})_{6}\right]$ complex remains stable at all three levels of theory and no cyanide dissociation occurs within the simulated time. From this data it can be concluded that counter-ions are essential for the observation of an octahedral cyanoferrate(II) complex in aqueous solution as reported in the literature. Therefore, it can be assumed that experi- 
mentally reported $\left[\mathrm{Fe}(\mathrm{CN})_{6}\right]^{4-}$ species in aqueous solution are stabilized by the respective cation of the dissolved salt.
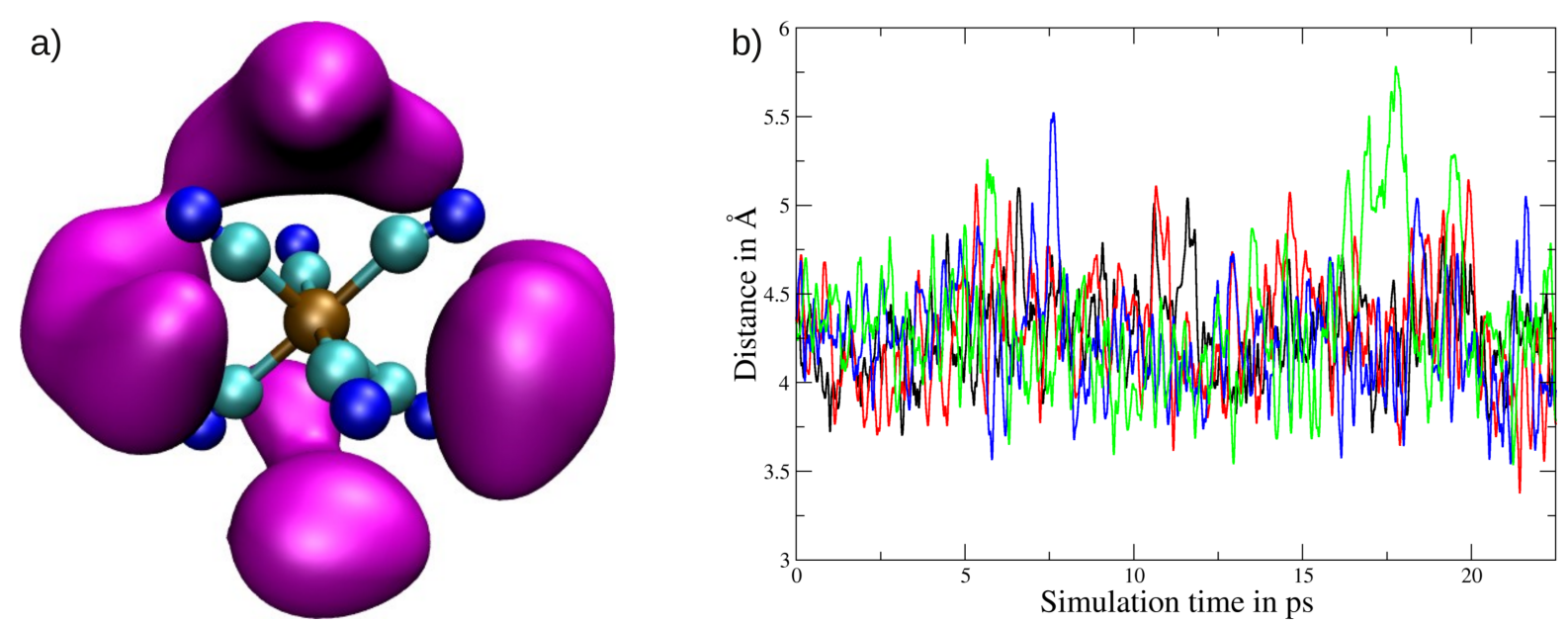

Figure 4: a) The hexacyanoferrate(II) complex with surrounding potassium counter-ions, which are depicted in form of a volume map projection. The potassium cations are highly mobile and are surrounding like clouds most of the complex. b) Fe-K distance plots: A large range of 3.5-5.8 $\AA$ is occupied by the stabilizing potassium ions. The four colours correspond to the four potassium ions circulating the complex.

Fig.5a shows Fe-C RDFs applying HF (black), B3LYP (red) and RI-MP2 (blue), respectively. An average bond distance of $2.16 \AA$ is obtained by HF, whereas bond distances of $1.94 \AA$ and $1.92 \AA$ are obtained from the respective MD simulations at the B3LYP and RI-MP2 level. The latter Fe-C bond distances are in full agreement with EXAFS data in aqueous solution, Table S2, proving our theoretically obtained data to be in excellent agreement with the experiment, in particular the data obtained from the RI-MP2 MD simulation. HF deviates as expected from the experimental values, and the reasons have been already discussed in the previous section. Fig.5b depicts Fe-N RDFs at all three levels of theory. Average bond distances of $3.30 \AA, 3.11 \AA$ and $3.07 \AA$ are reported using HF, B3LYP and RI-MP2, respectively. From the EXAFS experiment an average bond distance of $3.08 \AA$ was obtained. The values found in the literature range from 3.06 to $3.13 \AA$. Also in this case an excellent agreement of theoretical and experimental data can be stated. Fig.5c shows Fe-K RDFs at the considered levels of theory. The average distances are $4.17 \AA$ in case of HF, $4.00 \AA$ in case of B3LYP and $3.87 \AA$ in the RI-MP2 simulation. However, it is seen that the RDFs are broad indicating significant variations of the individual distance and hence confirms the rapid relocation of potassium ions surrounding the hexacyanoferrate(II) complex as depicted in Fig.4. The Fe-K distance range in solid 
$\mathrm{K}_{4}\left[\mathrm{Fe}(\mathrm{CN})_{6}\right]{ }^{66} 4.20-4.35 \AA$, is not directly comparable to our data, but can be seen as an indication that the location of the counter-ions in solution is similar as in the solid phase, but with much larger mobility. Fig. 5d shows the Fe-O RDFs at the three levels of theory (continuous lines) and the respective integration (dashed lines). A distinct hydration shell between 4 and $7 \AA$ is observed in all three cases, although the peak maximum is slightly shifted in case of HF compared to B3LYP and RI-MP2. Also an ordering influence of the potassium ions on the bulk is observed. The integration over the first hydration shell yields a number of 23-27 water molecules surrounding the complex. It is interesting that water molecules are not found at distances below $4 \AA$, as at this distance the majority of the potassium ions are located. This is emphasized in Fig.5e, where the trajectory of all four potassium ions obtained from the simulations is schematically depicted. Looking at the dense distribution of potassium ions around the complex it is understandable that most water molecules are prevented from coordinating closer to the hexacyanoferrate(II) complex.

However, from the N-H RDFs in fig.6a (continuous lines) it is seen that some water molecules coordinate to the nitrogen of the cyanide ligands. An average $\mathrm{N}-\mathrm{H}$ distance of $2.12 \AA$ (HF), $1.89 \AA$ (B3LYP) and $1.92 \AA$ (RI-MP2) is obtained, which is the typical distance for a hydrogen bond. From the integration (dashed lines) of the first peak an average number between two and three water ligands is obtained. The lifetime of the formed hydrogen bonds was evaluated by so called hydrogen bond correlation functions, ${ }^{69}$ which are depicted in Fig.6b. Since such correlation functions typically have a doubleexponential decay, the can be fitted using the following expression:

$$
y=a \cdot e^{-\frac{t}{\tau_{l}}}+(1-a) \cdot e^{-\frac{t}{\tau_{s}}}
$$

with $\tau_{l}$ and $\tau_{s}$ corresponding to the long range and short range contributions. The respective long range contribution $\tau_{l}$ is of particular interest, because it is a direct measure for the hydrogen bond lifetime. From the fitted correlation function (continuous line) an average hydrogen bond lifetime between $0.2 \mathrm{ps}(\mathrm{HF})$ and $0.3 \mathrm{ps}$ (B3LYP) is obtained. No data from the RI-MP2 simulations are reported because of the large time step of $2.0 \mathrm{fs}$ applied in the respective simulation. Nevertheless, it can be concluded that B3LYP assigns hydrogen bonds a higher stability compared to HF, which is a commonly known problem of DFT. However, a hydrogen bond lifetime between 0.2 ps and 0.3 ps is a clear hint of extremely fast dynamics indicating high water exchange rates similar or higher than in bulk water probably caused by the circulating potassium ions. 

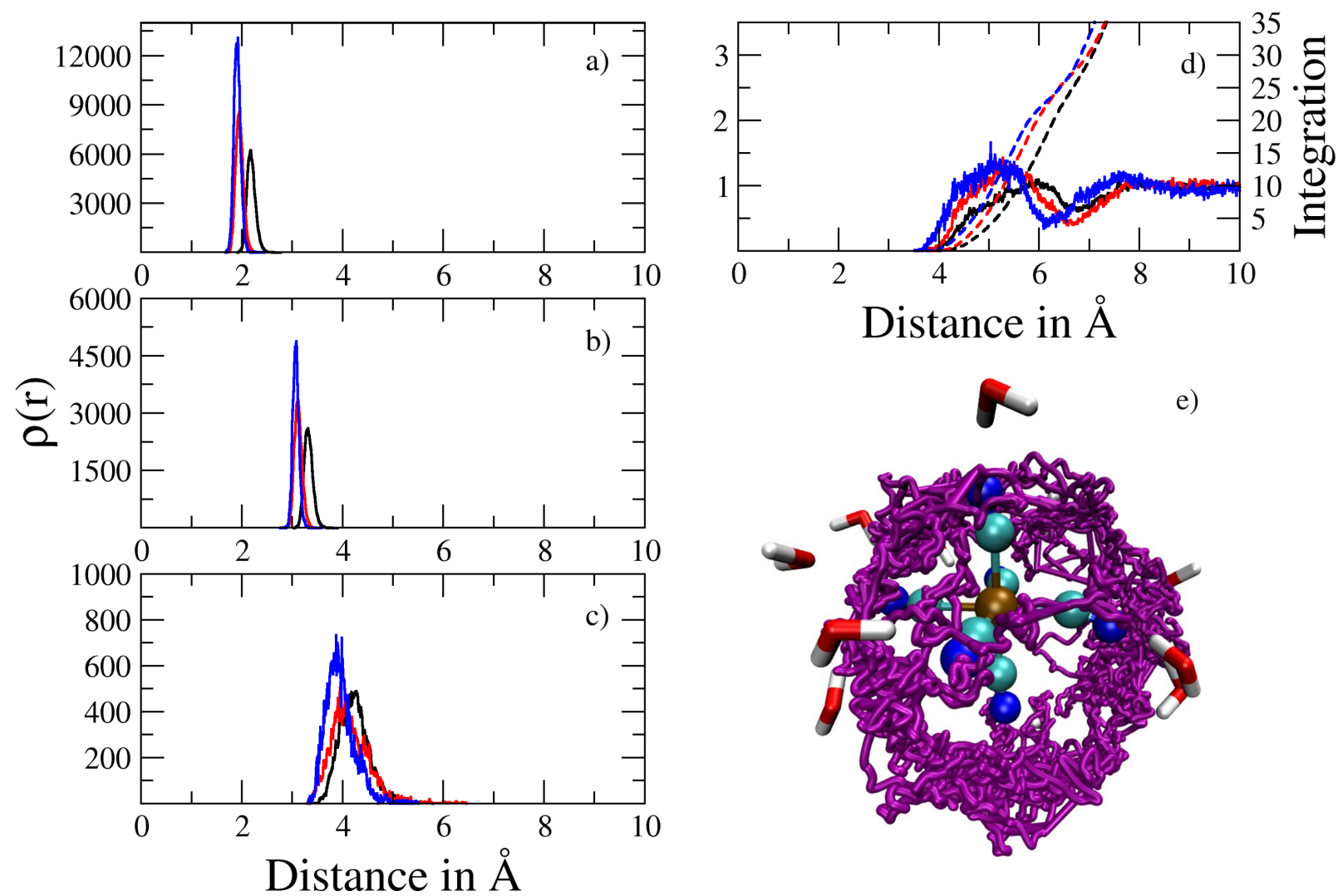

Figure 5: Radial distribution functions (RDFs) for $\mathrm{K}_{4}\left[\mathrm{Fe}(\mathrm{CN})_{6}\right]$ obtained from QMCF MD simulations at different levels of theory. Black curves denote RDFs obtained from simulations applying HF, red and blue curves denote the corresponding RDFs at the B3LYP and RI-MP2 level. a) Fe-C RDF b) Fe-N RDF c) Fe-K RDF d) Fe-O RDF and the respective integration over the peaks in dashed lines. e) Schematic illustration of the trajectory of the four potassium ions surrounding the hexacyanoferrate(II) complex. Most water molecules are prevented from entering the coordination sphere of iron(II). 

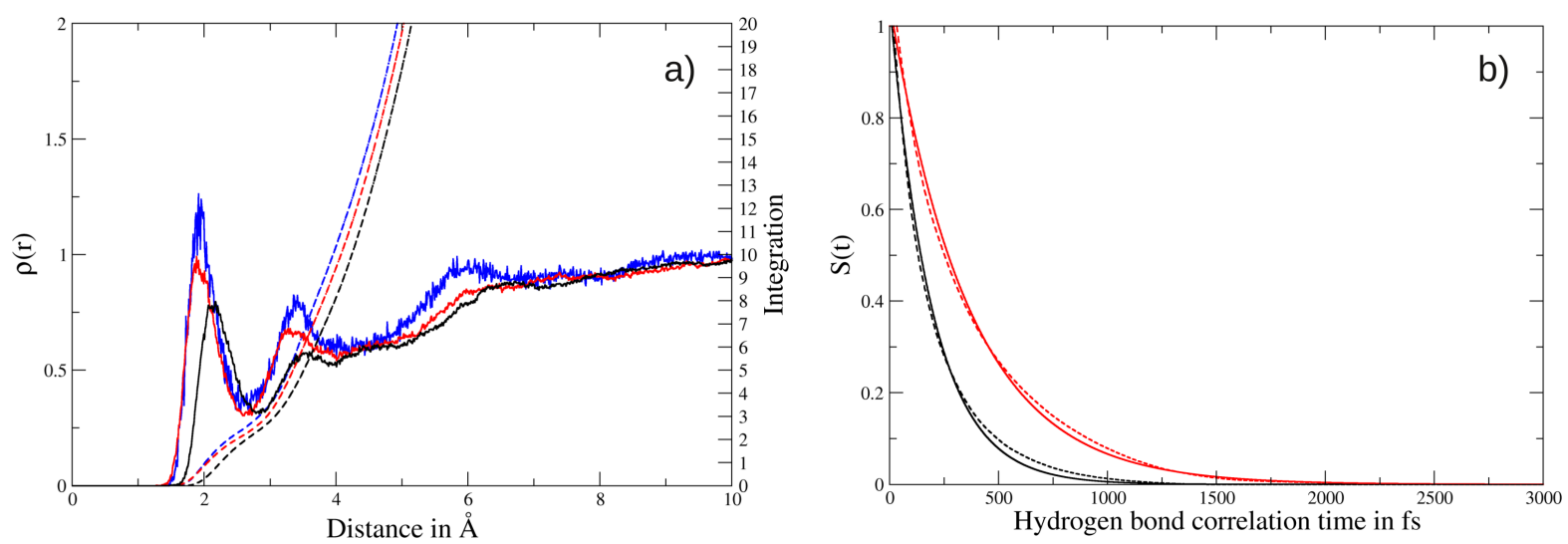

Figure 6: a) N-H RDFs at the HF (black), B3LYP (red) and RI-MP2 (blue) level of theory. b) Hydrogen bond correlation functions obtained from the HF (black continuous line) and B3LYP (red continuous line) simulation and the double-exponential fit (dashed lines).

\section{Conclusions}

Theoretical simulations of the hexacyanoferrate(II) ion, $\left[\mathrm{Fe}(\mathrm{CN})_{6}\right]^{4-}$, in aqueous solution on QMCF level have clearly shown that sole the highly negatively charged $\left[\mathrm{Fe}(\mathrm{CN})_{6}\right]^{4-}$ complex is not stable in aqueous solution and dissociates within picoseconds to a more stable complex with lower charge, $\left[\mathrm{Fe}(\mathrm{CN})_{4} \mathrm{H}_{2} \mathrm{O}\right]^{2-}$. The $\left[\mathrm{Fe}(\mathrm{CN})_{6}\right]^{4-}$ complex is on the other hand stable in presence of counter ions which surround the complex to significantly compensate the high charge through ion-pair formation. This has been proven by both theoretical simulations and experiments. The structure of the $\left[\mathrm{Fe}(\mathrm{CN})_{6}\right]^{4-}$ complex, surrounded by potassium ions, which are clearly seen in the simulation but not observable by experimental methods in solution, is identical in solid state and aqueous solution.

\section{Acknowledgements}

Financial support for this work by a $\mathrm{PhD}$ scholarship of the Leopold-Franzens-University of Innsbruck (Rector Prof. Dr.Dr.hc.mult. Tilmann Märk) for A.O. Tirler is gratefully acknowledged. This work was supported by the Austrian Ministry of Science BMWF UniInfrastrukturprogramm as part of the Research Focal Point Scientific Computing at the University of Innsbruck. 


\section{References}

[1] Ferlay, S.; Mallah, T.; Ouahes, R.; Veillet, P.; Verdaguer, M. Nature 1995, 378, 701703.

[2] Sato, O.; Iyoda, A.; Fujishima, A.; Hashimoto, K. Science 1996, 271, 49-51.

[3] Sato, O.; Iyoda, A.; Fujishima, A.; Hashimoto, K. Science 1996, 272, 704-705.

[4] Kumar, A.; Yusuf, S.; Keller, L.; Yakhmi, J.; Srivastava, J.; Paulose, P. Phys. Rev. B 2007, 75, 224419.

[5] Bleuzen, A.; Lomenech, C.; Escax, V.; Villain, F.; Varret, F.; Cartier dit Moulin, C.; Verdaguer, M. J. Am. Chem. Soc. 2000, 122, 6648-6658.

[6] Giorgetti, M.; Scavetta, E.; Berrettoni, M.; Tonelli, D. Analyst 2001, 126, 2186-2171.

[7] Scholz, F.; Dostal, A. Angew. Chem., Int. Ed. Engl. 1995, 34, 2685-2687.

[8] Coon, D.; Amos, L.; Bocarsly, A. Anal. Chem. 1998, 70, 3137-3145.

[9] Karyakin, A.; Kotel'nikova, E.; Lukachova, L.; Karyakina, E. Anal. Chem 2002, 74, $1597-1603$.

[10] Pyrasch, M.; Toutianoush, A.; Jin, W.; Schnepf, J.; Tieke, B. Chem. Mater. 2003, $15,245-254$.

[11] Zhou, D.; Ju, H.; Chen, X. J. Electroanal. Chem. 1998, 408, 219.

[12] Deng, Z.; Smyrl, W. J. Electrochem. Soc. 1991, 138, 1911-1918.

[13] Neff, V. J. Electrochem. Soc. 1985, 132, 1382-1384.

[14] Rader, W.; Solujić, L.; Milosavijević, E.; Hendrix, J. Env. Sci. Techn. 1993, 27, 18751879.

[15] Kolthoff, I.; Pearson, E. J. Ind. Eng. Chem. 1931, 3, 381-382.

[16] Giorgetti, M.; Della Longa, S.; Benfatto, M. J. Phys.: Conf. Ser. 2009, 190, 012145. 
[17] Giorgetti, M.; Berrettoni, M. Inorg. Chem. 2008, 47, 6001-6008.

[18] Hayakawa, K.; Hatada, K.; D’Angelo, P.; Della Longa, S.; Natoli, C.; Benfatto, M. J. Am. Chem. Soc. 2004, 126, 15618-15623.

[19] Meeussen, J.; Keizer, M.; de Haan, F. Env. Sci. Techn. 1992, 26, 511-516.

[20] Matsumara, M.; Kojima, T. J. Hazard. Mater. 2003, B97, 99-110.

[21] Olabe, J.; Zerga, H. Inorg. Chem. 1983, 22, 4156-4158.

[22] Nilchi, A.; Malek, B.; Maragheh, M.; Khanchi, A. J. Radioanal. Nucl. Chem. 2003, 258, 457-462.

[23] MacDiarmid, A.; Hall, N. J. Am. Chem. Soc. 1954, 76, 4222-4228.

[24] Naik, R.; Tiwari, R.; Yadav, S.; Kumar, B.; Asthana, A. Indian J. Chem. Technol. 2012, 19, 250-256.

[25] Sando, G.; Zhong, Q.; Owrutsky, J. J. Chem. Phys. 2004, 121, 2158-2168.

[26] Yu, Q.; Steen, W.; Jeerage, K.; Jiang, S.; Schwartz, D. J. Electrochem. Soc. 2002, 149, E195-E203.

[27] Szacilowski, K.; Stasicka, Z. Coord. Chem. rev. 2002, 229, 17-26.

[28] Prasad, S. Anal. Lett. 2004, 37, 2851-2867.

[29] Prampolini, G.; Yu, P.; Pizzanelli, S.; Cacelli, I.; Yang, F.; Zhao, J.; Wang, J. J. Phys. Chem. B 2014, 118, 14899-14912.

[30] Engel, N.; Bokarev, S.; Suljoti, E.; Garcia-Diez, R.; Lange, K.; Atak, K.; Golnak, R.; Kothe, A.; Dantz, M.; Kühn, O.; Aziz, E. J. Phys. Chem. B 2014, 118, 1555-1563.

[31] Yu, P.; Yang, F.; Zaho, J.; Wang, J. J. Phys. Chem. B 2014, 118, 3104-3114.

[32] Basolo, F. Coord. Chem. Rev. 1968, 3, 213-223.

[33] Bühl, M.; Schreckenbach, G.; Sieffert, N.; Wipff, G. Inorg. Chem. 2009, 48, 99779979.

[34] Goedken, V.; Vallarino, L.; Quagliano, J. J. Am. Chem. Soc. 1970, 92, 303-307. 
[35] Tirler, A.; Hofer, T. J. Phys. Chem. B 2014, 118, 12938-12951.

[36] Warshel, A.; Levitt, M. J. Mol. Biol. 1976, 103, 227-249.

[37] Rode, B.; Hofer, T.; Randolf, B.; Schwenk, C.; Xenides, D.; Vchirawongkwin, V. Theor. Chem. Acc. 2006, 115, 77-85.

[38] Rode, B.; Hofer, T. Pure Appl. Chem. 2006, 78, 525-539.

[39] Rode, B.; Hofer, T.; Pribil, A.; Randolf, B. Advances in Inorganic Chemistry; Elsevier, 2010; Vol. 62; Chapter 4, pp 143-175.

[40] Rode, B.; Hofer, T.; Pribil, A.; Randolf, B. Advances in Quantum Chemistry; Elsevier, 2010; Vol. 59; Chapter 7, pp 213-246.

[41] Tirler, A.; Weiss, A.; Hofer, T. J. Phys. Chem. B 2013, 117, 16174-16187.

[42] Lutz, O.; Messner, C.; Hofer, T.; Glaetzle, M.; Huck, C.; Bonn, G.; Rode, B. J. Phys. Chem. Lett. 2013, 4, 1502-1506.

[43] Messner, C.; Lutz, O.; Rainer, M.; Huck, C.; Hofer, T.; Rode, B.; Bonn, G. J. Phys. Chem. B 2014, 118, 12232-12238.

[44] Tarique, M.; Hofer, T. Mol. BioSyst. 2014, 10, 117-127.

[45] Feyereisen, M.; Fitzgerald, G.; Komornicki, A. Chem. Phys. Lett. 1993, 208, 359-363.

[46] Vahtras, O.; Almlöf, J.; Feyereisen, M. Chem. Phys. Lett. 1993, 213, 514-518.

[47] Bernholdt, D.; Harrison, R. Chem. Phys. Lett. 1996, 250, 477-484.

[48] Berendsen, H.; Postma, J.; van Gunsteren, W.; DiNola, A.; Haak, J. J. Chem. Phys. 1984, 81, 3684-3690.

[49] Andersen, H. J. Chem. Phys. 1980, 72, 2384-2393.

[50] Barker, J.; Watts, R. Mol. Phys. 1973, 26, 789-792.

[51] Watts, R. Mol. Phys. 1974, 28, 1069-1083.

[52] Dolg, M.; Wedig, U.; Stoll, H.; Preuss, H. J. Chem. Phys. 1987, 86, 866-872.

[53] Hariharan, P.; Pople, J. Theoret. Chim. Acta 1973, 28, 213-222. 
[54] Rassolov, V.; Pople, J.; Ratner, M.; Windus, T. J. Chem. Phys. 1998, 109, 1223-1229.

[55] Krishnan, R.; Binkley, J.; Seeger, R.; Pople, J. J. Chem. Phys. 1980, 72, 650-654.

[56] Blaudeau, J.-P.; McGrath, M.; Curtiss, L.; Radom, L. J. Chem. Phys. 1997, 10\%, 5016-5021.

[57] Liew, C.; Inomata, H.; Arai, K. Fluid Phase Equil. 1998, 144, 287-298.

[58] Kräutler, V.; van Gunsteren, W.; Hünenberger, P. J. Comput. Chem. 2001, 22, 501508.

[59] Reif, M.; Hünenberger, P. J. Chem. Phys. 2011, 134, 144104.

[60] Thompson, A.; Attwood, D.; Gullikson, E.; Howells, M.; Kim, K.-J.; Kortright, J.; Lindau, I.; Pianatta, P.; Robinson, A.; Scofield, J.; Underwood, J.; Vaughan, D.; Williams, G.; Winick, H. X-Ray Data Booklet; LBNL/PUB-490 Rev.2, Lawrence Berkeley National Laboratory, Berkeley, California 94720, 2001.

[61] George, G.; Pickering, I. EXAFSPAK-A Suite of Computer Programs for Analyis of X-Ray Absorption Spectra; SSRL: Stanford, Ca, 1993.

[62] Sayers, D.; Bunker, B. In X-Ray Absorption Principles, Applications and Techniques of EXAFS, SEXAFS and XANES; Koningsberger, D., Prins, R., Eds.; WileyInterscience, New York,, 1988; Chapter 6.

[63] Szabinsky, S.; Rehr, J.; Ankudinov, A.; Albers, R.; Eller, M. Phys. Rev. B 1995, 52, 2995-3009.

[64] Marcus, Y. Chem. Rev. 2009, 109, 1346-1370.

[65] Marcus, Y. J. Solution Chem. 1994, 23, 831-848.

[66] Willans, M.; Wasylishen, R.; McDonald, R. Inorg. Chem. 2009, 48, 4342-4353.

[67] Taylor, J.; Mueller, M.; Hitterman, R. Acta Crystallogr. A 1970, 26, 559-567.

[68] Pospelov, V.; Zhdanov, G. Zh. Fiz. Khim. 1947, 21, 405-406.

[69] Chanda, J.; Chakraborty, S.; Bandyopadhyay, S. J. Phys. Chem. B 2006, 110, 37913797. 
For Table of Contents Only
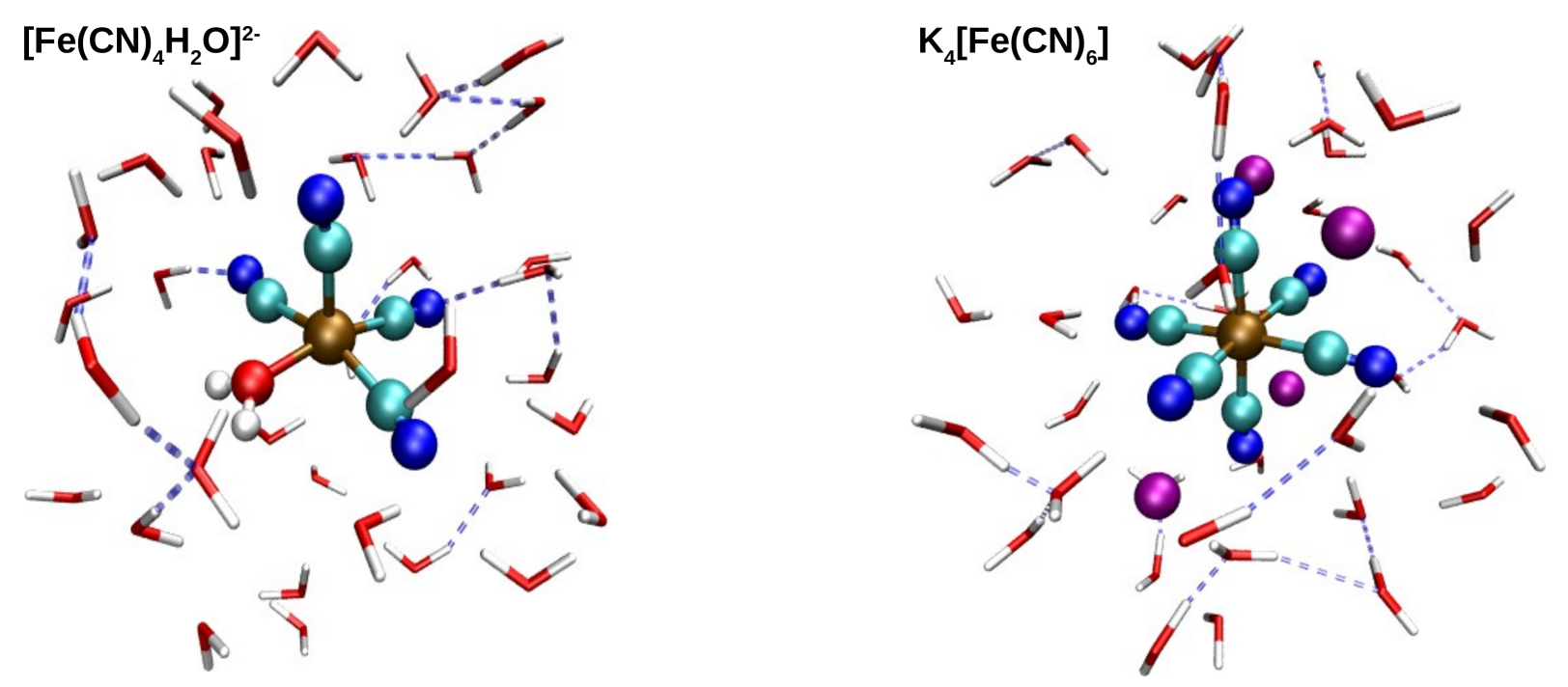